\title{
Double Bragg grating tunable filter
}

Sebastien Blais-Ouellette, Edward H. Wishnow, Patrick L Shopbell, Wil van Breugel, Keith Taylor, et al.

Sebastien Blais-Ouellette, Edward H. Wishnow, Patrick L Shopbell, Wil van Breugel, Keith Taylor, Roger Smith, "Double Bragg grating tunable filter," Proc. SPIE 5492, Ground-based Instrumentation for Astronomy, (30 September 2004); doi: 10.1117/12.551743

Event: SPIE Astronomical Telescopes + Instrumentation, 2004, Glasgow, United Kingdom 


\title{
Double Bragg Grating Tunable Filter
}

\author{
Sébastien Blais-Ouellette ${ }^{a, b}$, Edward H. Wishnow ${ }^{c}$, Patrick L. Shopbell ${ }^{b}$, Wil van Breugel ${ }^{c}$, \\ Keith Taylor ${ }^{b}$ and Roger Smith $^{b}$ \\ ${ }^{a}$ Photon etc. inc., 432 boul. des Prairies, Laval, Canada \\ ${ }^{b}$ California Institute of Technology, 1200 E. California blvd., Pasadena, CA, USA \\ ${ }^{c}$ Lawrence Livermore National Laboratory, 7000 East Ave, Livermore, USA
}

\begin{abstract}
A novel concept for a very efficient tunable filter for the optical and near infrared is proposed. The filter consists of two parallel volume phase holographic Bragg gratings used in a dispersion-recombination configuration. The passband profile is determined by the first order grating efficiency curve which changes in wavelength according to the angle of incidence. The filter can be continuously tuned over ranges of wavelengths larger than $600 \mathrm{~nm}$ by adjusting the tilt of the gratings, while bandwidth can be selected between 0.1 and $200 \mathrm{~nm}$. The conceptual design is presented, along with predicted performances. Potentially useful instruments for large volume surveys and targeted observations are explored, along with application in areas other than astronomy.
\end{abstract}

Keywords: astronomy, instruments, near infrared, visible, tunable filter

\section{INTRODUCTION}

Narrow band imaging is a key ingredient of observational astronomy. The capacity to isolate a known spectral feature allows a deep view of our universe by removing unwanted photons from the night sky and from the source of interest itself. Until recently, narrow band imaging almost exclusively used fixed band filters, except for the highest resolution, where Fabry-Perot etalons were, and still are, very capable. The fixed nature of most filters restricts them to known lines at known redshifts. In the last decade, considerable efforts have been made to develop tunable filters that allow the observation of arbitrary spectral features.

Among the most successful astronomical tunable filter is the Fabry-Perot etalon-based Taurus Tunable Filter on the Anglo-Australian Telescope. ${ }^{1}$ Etalon-based filters are adjustable both in wavelength and bandpass, but they are limited to a very narrow band, around $2 \mathrm{~nm}$ at $1 \mu \mathrm{m}$, without endangering the plate coatings. Also, the complexity is increased and the efficiency is impaired by the necessity to use order sorting filters. Other concepts including acousto-optics or Lyot filters ${ }^{2}$ are either limited in tunability, or in throughput, or they are optically very complex.

We propose a novel concept based on the intrinsic angular dependence of Bragg diffraction efficiency. Using two parallel identical gratings sequentially, in a dispersion-recombination configuration, it is possible to design an imaging system with adjustable passband and efficiency peaking above $85 \%$.

\section{CONCEPTUAL DESIGN}

One of the most interesting features of Volume Phase Holographic (VPH) gratings is the possibility of adjusting the efficiency curve, or "blaze" function, by varying the angle of incidence. Indeed, while their dispersive properties are identical to that of classical gratings, their diffracted energy distribution is governed by Bragg's law, as for X-rays in a crystalline structure. In the case where the modulation fringes are perpendicular to the grating surface, the Bragg condition follows,

$$
m \nu \lambda=2 n_{\text {grating }} \sin \alpha_{\text {grating }}
$$

Send correspondence to sbo@photonetc.com 


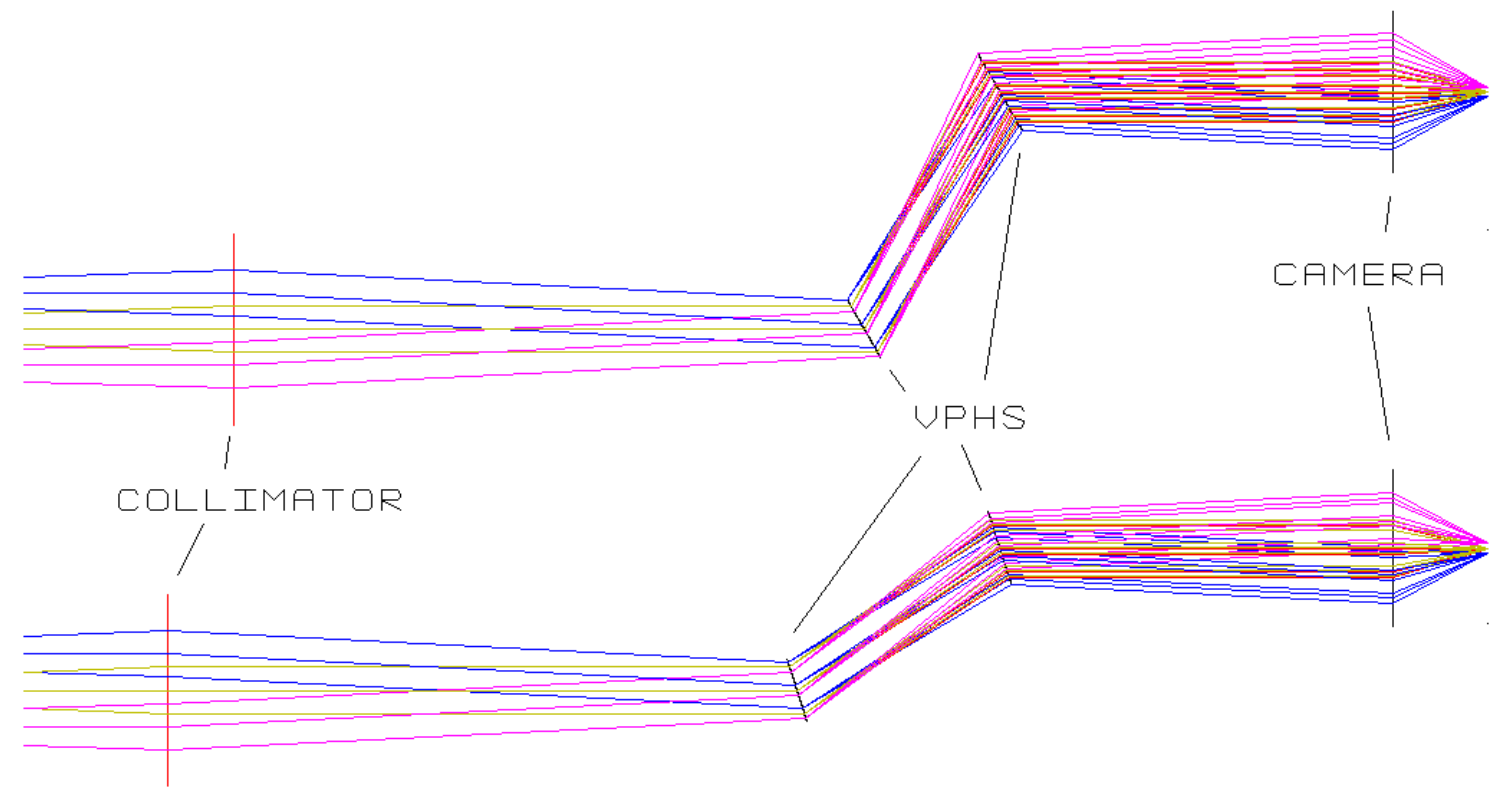

Figure 1. Optical layout of the Double VPH Tunable Filter. A first grating disperses collimated light that satisfies the Bragg condition. A second grating recombines the beam which is then re-imaged onto a detector. Top: grating angle is 30 , passband is centered on 500nm for a 2000 lines/mm VPH. Bottom: grating angle is 17.5, passband is centered 300 $\mathrm{nm}$ for the same VPHs.

and from Snell's law

$$
m \nu \lambda=2 \sin \alpha_{\text {air }}
$$

where $\mathrm{m}$ is the order of diffraction, $\nu$ is the fringe frequency in the grating, $\lambda$ is the wavelength, $\mathrm{n}$ is the average index of refraction, and $\alpha$ is the angle of incidence. Radiation that departs significantly from the Bragg condition passes through the grating undiffracted. This tunability can be advantageously used in spectrographs ${ }^{3}$ but it also allows a new type of imaging tunable filter.

Using a second grating, it is possible to recombine, or "undisperse", the light coming from the first grating. An image can be reconstructed as long as the gratings are parallel and have the same line fringe frequency. The Double Bragg Grating Tunable Filter (DBGTF) is illustrated in Fig. 1. Only light whose wavelength satisfies the Bragg condition is diffracted. It is then possible to adjust the grating angle, effectively tuning the filter central wavelength.

Our current design tries to balance off-axis angles and collimator size. The specific choice depends on the acceptable wavelength gradient across the field. For large wavelength-span survey modes, such gradients are not a handicap and a compact instrument can be designed. On the other hand, for targeted observation, one would want a quasi-monochromatic field, implying a long focal length and thus a large pupil system. Interestingly, current technology allows the building of very large format VPH gratings (up to half a meter). Such large pupil instruments could allow a low gradient over a reasonable field, even for $10 \mathrm{~m}$ class telescopes. It is also important to remember that the gradient is in the dispersion axis only. A very large field could be obtained with a rectangular detector, with its short axis aligned to the dispersion direction.

Another important aspect of the system is to ensure proper parallelism of the gratings while varying the angles over many degrees. Preliminary optical design studies show that parallelism must be maintained to less then an arcminute. Such precision can be easily obtained with current opto-mechanical technologies 


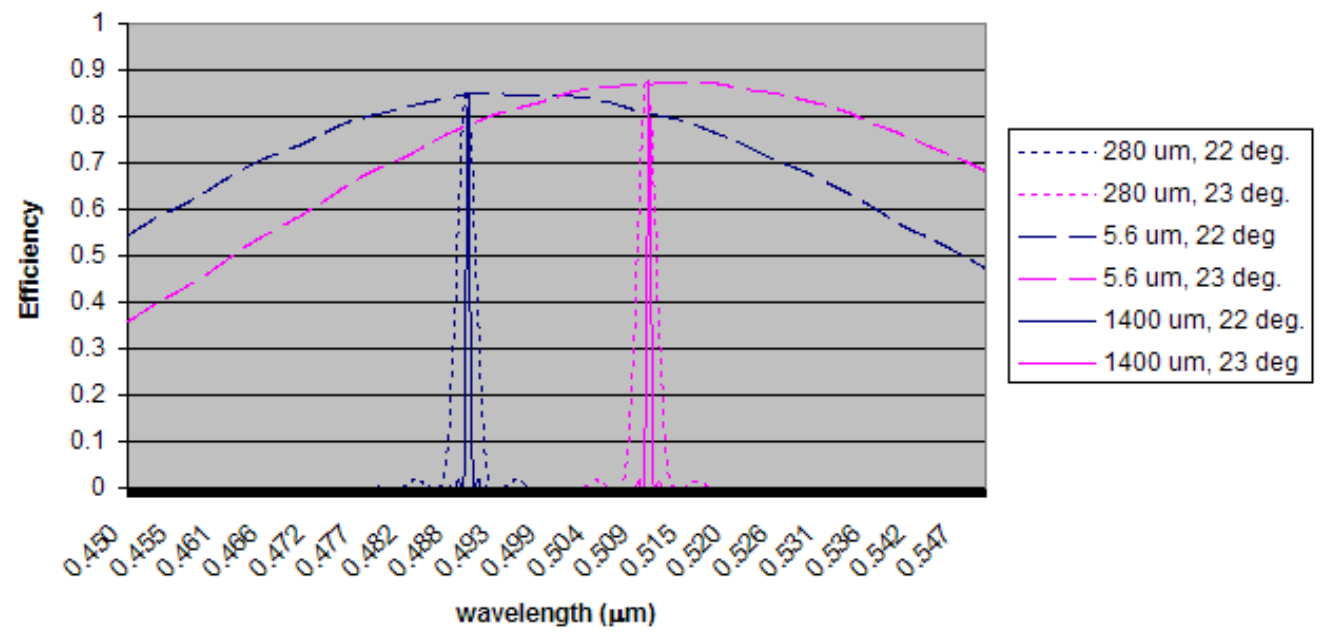

Figure 2. Efficiency curves for three different pairs of VPH. All are optimized for a wavelength of $0.5 \mathrm{~nm}$ at $22.5^{\circ}(\nu=1530$ $\mathrm{l} / \mathrm{mm}) . \Delta \lambda_{F W H M}$ are $0.5 \mathrm{~nm}, 2.5 \mathrm{~nm}$, and $112 \mathrm{~nm}$ for thicknesses of $1400 \mu \mathrm{m}, 280 \mu \mathrm{m}$, and $5.6 \mu \mathrm{m}$ respectively.

\section{EXPECTED PERFORMANCE}

According to Kogelnik's coupled wave analysis, ${ }^{4}$ efficiency of thick gratings with unslanted fringes is approximately, for the two planes of polarization:

$$
\begin{gathered}
\eta_{s}=\sin ^{2}\left(\frac{\pi \Delta n}{\lambda} \frac{d}{\cos \alpha}\right) \\
\eta_{p}=\sin ^{2}\left(\frac{\pi \Delta n \cos (2 \alpha)}{\lambda} \frac{d}{\cos \alpha}\right)
\end{gathered}
$$

where $\alpha$ is the angle of incidence in the air, $\nu$ is the fringe frequency, and $\mathrm{d}$ the depth of the grating structure. Also, the wavelength bandwidth is given by:

$$
\frac{\Delta \lambda_{F W H M}}{\lambda} \simeq \frac{\cot \alpha}{\nu d}
$$

In the context of an instrument for astronomy, it is more attractive to minimize the relative wavelength shift due to off-axis angles to keep the field-of-view as monochromatic as possible. One wants to minimize

$$
\frac{\partial \lambda}{\partial \alpha}=\frac{2 n \cos \alpha}{m \nu} \simeq \frac{2 \cos \alpha}{\nu}
$$

However, in order for p-plane efficiency not to differ significantly from s-plane efficiency, one must keep $\alpha \ll \frac{\pi}{4}$. Taking $30^{\circ}$ as a maximum grating angle, the Bragg condition limits $\nu$ to 2000 lines $/ \mathrm{mm}$ for a wavelength of 500 $\mathrm{nm}$ and the wavelength shift is thus close to $15 \mathrm{~nm}$ per degree $(\alpha)$.

More rigorous Gsolver* simulations confirm the possibility of obtaining bandwidths between $0.1 \mathrm{~nm}$ and $200 \mathrm{~nm}$, but fringing starts to appear for high grating thicknesses (narrow bandwidths). This may not be a problem if proper apodization is applied to the index modulation pattern, although this may impact efficiency somewhat. This apodization is routine in Bragg filters for optical fibers and is possible, according to manufacturers, for volume gratings as well. Figure 2 shows examples of $0.5,2.5$, and $112 \mathrm{~nm}$ bandwidth filters, tunable from $350 \mathrm{~nm}$ to $800 \mathrm{~nm}$ with efficiency above $50 \%$.

It is possible to optimize the peak efficiency to any desired wavelength region by adjusting thickness and modulation as shown in Fig.3, where peak efficiency over the whole tunable range is shown. It is clear from this example that the design parameter space is vast and trade-offs are complex and numerous.

\footnotetext{
${ }^{*}$ Grating Solver Development Company: www.gsolver.com
} 


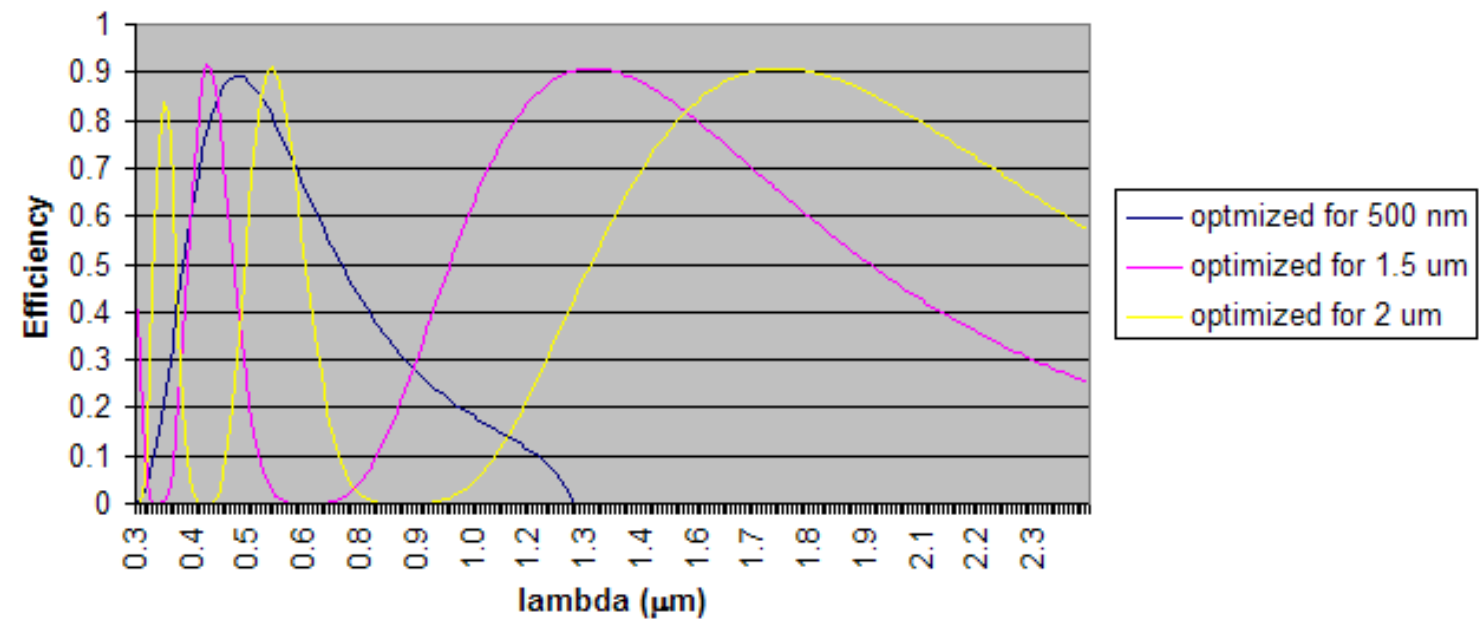

Figure 3. Peak diffracted energy efficiencies for three gratings optmized for wavelengths of $0.5,1.5$, and $2 \mu \mathrm{m}$.

\section{SCIENCE CASES}

The range of scientific applications is extensive. Many current observations made with conventional interference filters will be facilitated by the availability of a simple, versatile and efficient tunable filter. Rather than enumerating all such science cases, we focus on science projects that are essentially reliant on a tunable filter.

\subsection{Giant Emission Line Nebulae and Proto-Clusters}

Since the discovery of the first High Redshift Radio Galaxies, it has been known that many are surrounded by large emission-line nebulae. Because the nebulae are centered on large, young galaxies, they provide a unique opportunity to study galaxy formation, galaxy-intergalactic medium (IGM) feedback mechanisms, the evolution of the chemical enrichment of the universe, and other similar processes. Such nebulae have been found around radio galaxies (e.g. Figure $4^{5}$ ) and powerful quasars (e.g., MR 2251-178). ${ }^{6}$

Clusters of galaxies are also convenient targets for studies of the formation and evolution of galaxies and may help constrain important cosmological parameters. Optical and near-IR surveys, assisted by X-ray selection, have found clusters out to $\mathrm{z} \sim 1.3 .^{7,8}$ Very deep optical 'Lyman break' surveys have discovered galaxy overdensities ('proto-clusters') at $\mathrm{z} \sim 3 .^{9}$ Such observations are time consuming and increasingly difficult at higher redshifts. Surveys may not be able to provide substantial numbers of over-dense regions spread over a large range in redshift, which would be needed if one wishes to investigate how proto-clusters evolve, at what redshifts galaxy clusters become virialized, and what the cluster mass functions are.

A practical way to identify proto-clusters is to search for over-dense regions around targeted objects, using narrow-band imaging. At least some fraction of the young galaxies in proto-clusters can be expected to be active star forming systems and such $\mathrm{Ly}_{\alpha}$ excess galaxies (LaEG's) should be detectable in deep emission-line searches (rest-frame $\mathrm{Ly}_{\alpha}$ is observed at optical wavelengths for objects with $\mathrm{z}>2$ ). A deep narrow-band $\mathrm{Ly}_{\alpha}$ image of the $\mathrm{z}=3.09$ SSA22a proto-cluster field, which was initially found using broad-band color selection, has confirmed this technique. ${ }^{10}$

Emission-line imaging of the fields around targeted high redshift objects can push searches for galaxy protoclusters to much higher redshifts than would otherwise be possible. To fully exploit emission-line imaging as a cosmological tool will require large field-of-view tunable filters. Large $\left(>200 \mathrm{~cm}^{2}\right)$ narrow-band filters for the largest telescopes are expensive, even for modest fields of view $\left(273 \mathrm{~cm}^{2}\right.$ for a $81 \mathrm{arcmin}^{2}$ FOV in the case of DEIMOS at Keck for example). This problem will be even more acute for the next generation of extremely large telescopes such as the CELT 30m (now TMT). The proposed filter can be designed to possess a very large 


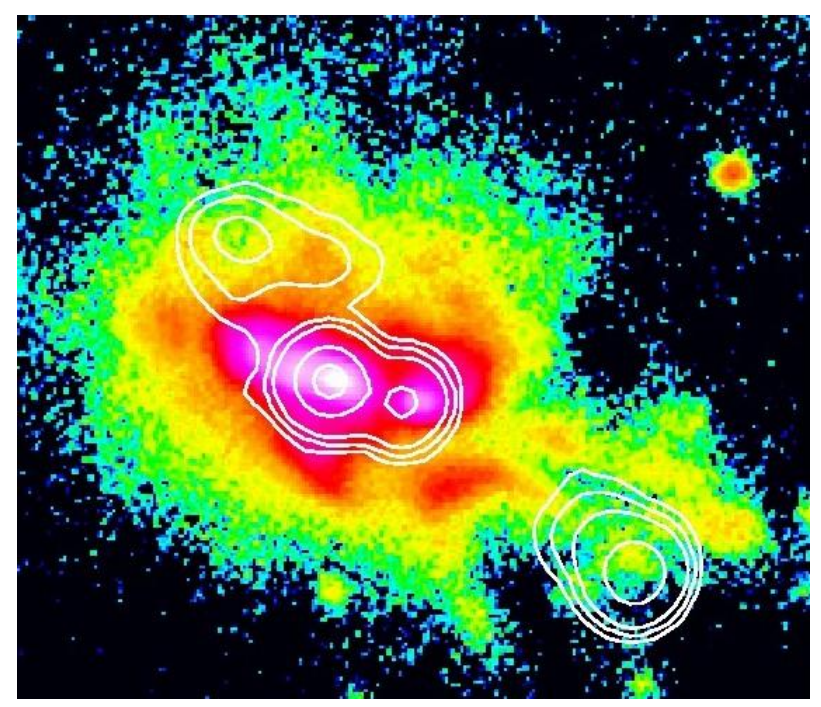

Figure 4. Keck narrow-band $\mathrm{Ly}_{\alpha}$ image (greyscale) of 4C41.17 overlaid with a VLA radio image at $6 \mathrm{~cm}$ (contours). The cross shows the position of the radio core.

quasi-monochromatic field-of-view. Indeed, contrary to other types of filters, there is no central wavelength shift in the non-dispersion axis. Moreover, spectral and spatial scanning can be used to remove the gradient in the other axis.

It is also clear that the selection of emission-line objects provides only partial insight into the galaxy populations and kinematics of proto-clusters, and obtaining spectroscopic absorption line redshifts of faint galaxies will be very difficult. The most powerful observational tool here would again be a tunable filter with medium sized (10-30nm wide) bandpasses so that accurate photometric redshift measurements can be made. The proposed double VPH tunable filter is perfectly matched to the task.

\subsection{Primeval galaxies}

Deep narrow band imaging enables the detection of primeval galaxies. A DBGTF will be a powerful tool for investigating galaxy formation, as already suggested by the detection of LEGOS (Ly ${ }_{\alpha}$ Emitting Galaxy-building ObjectS $)^{11}$ on the VLT: In a $7 \times 7$ arcmin field of view, with 10 hours exposure through a $2 \mathrm{~nm}$ FWHM filter in FORS1, they detect $35 \mathrm{Ly}_{\alpha}$ emitting galaxies (all in the same redshift range: $\mathrm{z}=2.85 \pm 0.03$ ). For a survey of this type, a tunable filter is virtually required, in order to obtain a statistically meaningful sample in the redshift domain.

Several groups are now pushing to $\mathrm{z}=5$ and beyond, also looking for the detection of $\mathrm{Ly}_{\alpha}$. The candidates are generally selected from multicolor imaging surveys such as the Sloan Digital Sky Survey or targeted narrowband imaging such as the Large-Area Lyman Alpha Survey ${ }^{12}$ which has recently confirmed three Ly $_{\alpha}$ emitters at $\mathrm{z}=$ 5.7. ${ }^{13}$ Also recently, $\mathrm{z} \sim 6$ quasars have been detected through a similar multi-band imaging and spectroscopic follow-up technique. These are being used to probe the nature of the Gunn-Peterson effect.

A truly efficient and wide-field tunable filter would allow such programs to scan regions of the sky at arbitrary redshift, increasing the potential candidates by an enormous factor. No other device can achieve the expected $75 \%$ average throughput, full optical band tunability, and a moderate to large field-of-view. Bandwidth can moreover be designed to match the application, providing an extremely versatile imaging device. 

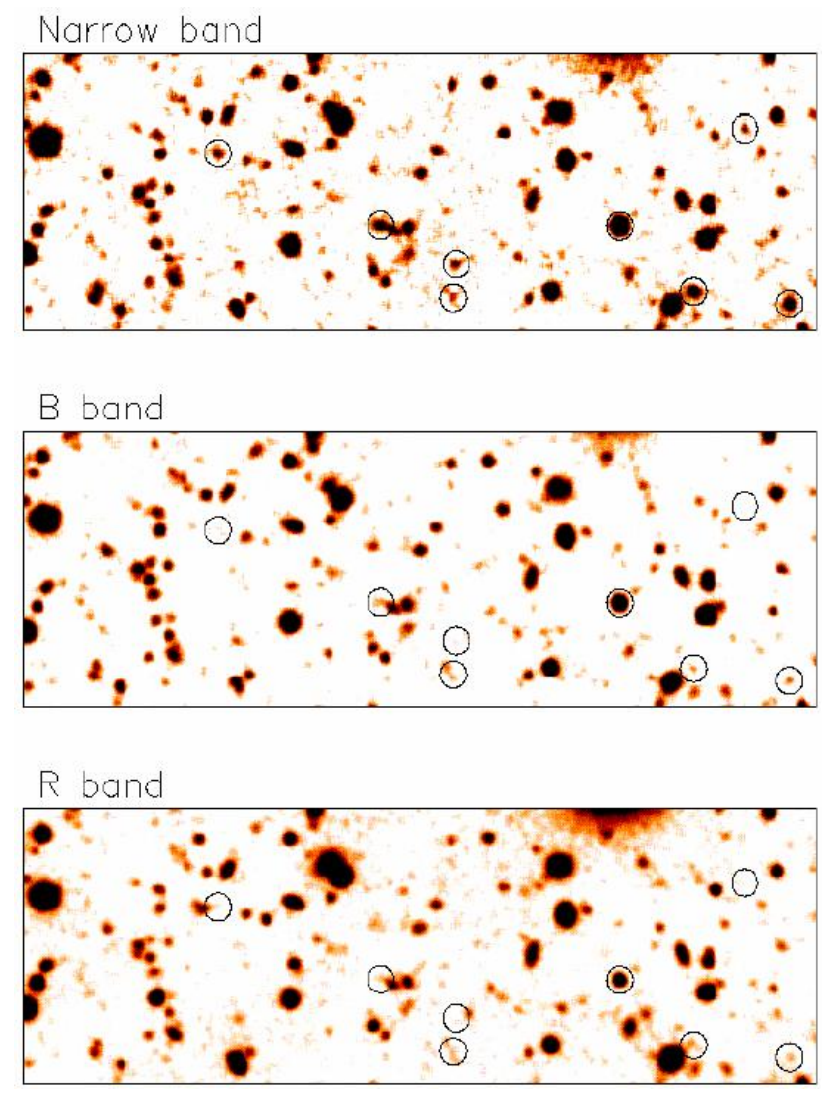

Figure 5. These images, by Fynbo et al. (2001) show the detection of Ly $\mathrm{y}_{\alpha}$ emitting galaxies at $\mathrm{z}=2.85 \pm 0.03$. The field of view is 40 " $\mathrm{x} 120$ " and the bandwidth $2 \mathrm{~nm}$.

\section{APPLICATIONS AND DESIGNS}

\subsection{Compact instrument}

Figure 1 shows the DBGTF configurations for two transmitted wavelengths. The gratings are parallel, but the input and output optical axes are translated with respect to each other. An imaging tunable filter can be more conveniently built if the camera is fixed relative to the input optics. A variety of optical designs allow for less movement of the output optics and can be optimized for specific applications. These designs can easily integrate a precise angular scanning mechanism to tune the transmitted wavelength.

\subsection{Wavelength Scanning}

A tunable filter can often be turned into a powerful imaging spectrometer if a proper scanning mechanism is provided and very narrow band gratings are used. Due to its large tunable range the DBGTF, can take full advantage of a precise scanning mechanism. Moreover, higher diffraction orders are naturally filtered angularly, removing the need for additional filtering optics.

Such an efficient and versatile imaging spectrograph can lead to a plethora of instrument concepts and applications. Astronomical instruments can be designed to have both a very narrow band for scanning imaging spectrometry, and a narrow to intermediate band for deep narrow band imaging at arbitrary wavelengths. Similar devices can be designed for earth observation and remote sensing where a fast, scanning narrow band imager is especially well adapted to the high level of light. This capacity to reject out-of-band light is a significant advantage over Fourier transform spectrographs, even more so considering that it can cover all the visible spectrum in addition to the the near-infrared. 

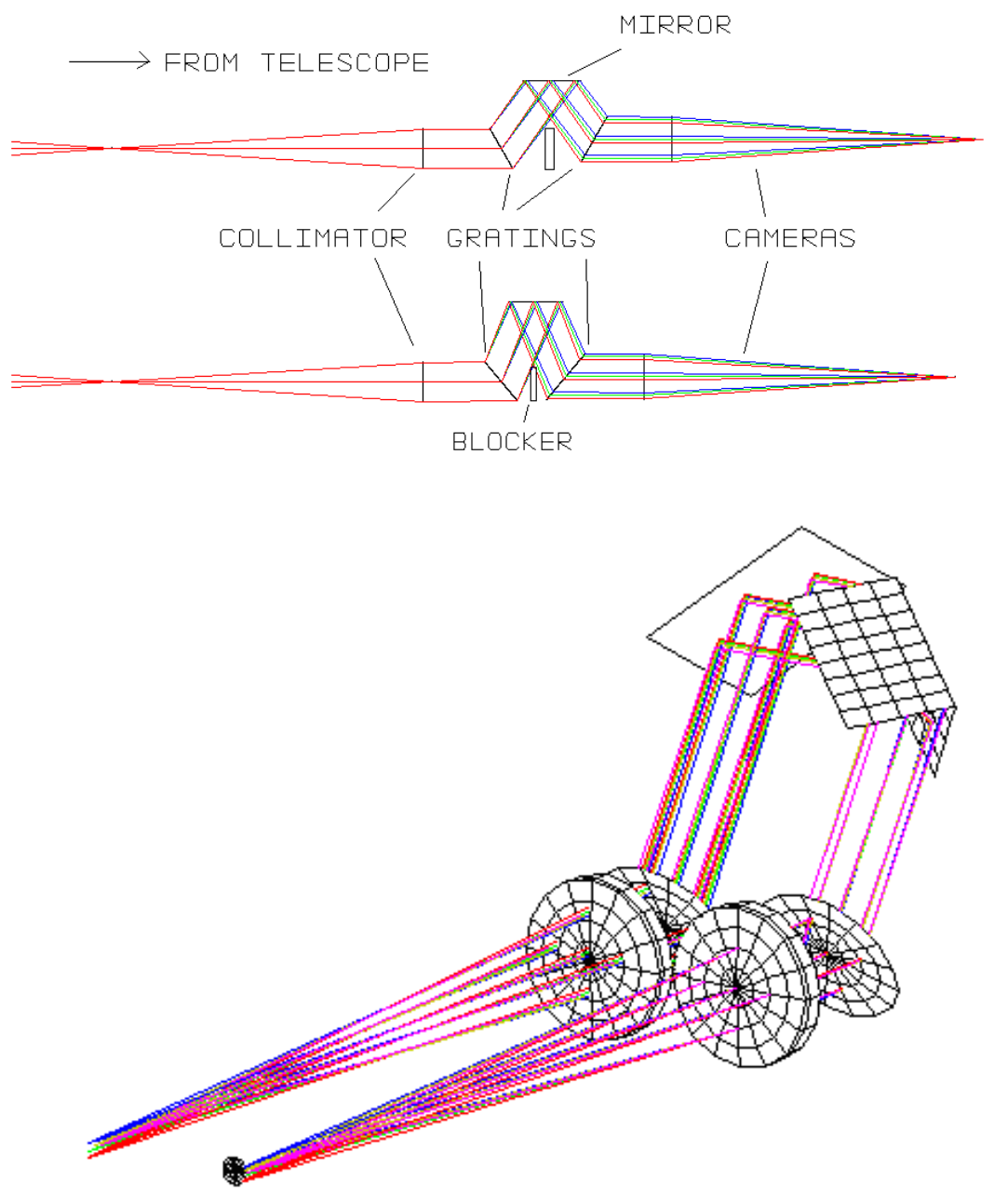

Figure 6. Upper drawings: The wavelength is tuned by symmetrically drawing the mirror, grating and camera optics closer to the input grating. The input and output optic axes remain aligned. Lower drawing: The input and output optics are fixed and the wavelength is tuned by changing the angle of the grating pair and the retro-reflector assembly. Light is introduced at the right side of the system and imaged at the left.

Microscopy is another natural field of application for the DBGTF. The device can be tuned to provide images over a set of fluorescence lines where normally this requires imaging through a set of fixed narrow band filters. When new fluorescent labels are developed, a new filter need not be purchased, but the DBGTF only needs to be moved to a new position. In addition, Raman microscopy is made possible by using two DBGTFs in series. The first filter can be used to remove the laser excitation line and the second to scan the Raman spectrum. In so doing, one can obtain a full spectral data-cube of the Raman emission of the sample.

\section{CONCLUSION}

The Double Bragg Grating Tunable Filter presented here has an efficiency reaching $85 \%$ for a bandwidth chosen between $1 \AA$ and $200 \mathrm{~nm}$. The central wavelength is tunable over a range of $600 \mathrm{~nm}$ with reasonable efficiency. This new concept opens exciting possibilities for astronomical narrow to intermediate band observation as well as for integral field spectroscopy. 
The tunable filter can naturally be integrated into other imaging applications such as remote sensing, Raman microscopy, as well as telecommunication and laboratory spectroscopy. In all cases, performances rely on high quality narrow band holographic gratings and a high precision scanning mechanism.

\section{ACKNOWLEDGMENTS}

The work by EW and WvB was performed under the auspices of the U.S. Department of Energy by the University of California, Lawrence Livermore National Laboratory under contract No. W-7405-Eng-48.

\section{REFERENCES}

1. J. Bland-Hawthorn and D. H. Jones, "Taurus Tunable Filter: A flexible approach to narrowband imaging," Publications of the Astronomical Society of Australia 15, pp. 44-49, Apr. 1998.

2. J. Bland-Hawthorn, W. van Breugel, P. R. Gillingham, I. K. Baldry, and D. H. Jones, "A Tunable Lyot Filter at Prime Focus: a Method for Tracing Supercluster Scales at z 1," ApJ 563, pp. 611-628, Dec. 2001.

3. S. C. Barden, J. B. Williams, J. A. Arns, and W. S. Colburn, "Tunable Gratings: Imaging the Universe in 3-D with Volume-Phase Holographic Gratings (Review)," in ASP Conf. Ser. 195: Imaging the Universe in Three Dimensions, pp. 552-+, 2000.

4. H. Kogelnik, "Coupled wave theory for thick hologram gratings," Bell System Tech. J. 48(9), p. 2909, 1969.

5. W. J. van Breugel, M. A. Reuland, W. H. de Vries, A. Stanford, A. Dey, J. Kurk, B. Venemans, H. J. A. Roettgering, G. Miley, C. De Breuck, M. Dopita, R. Sutherland, and J. Bland-Hawthorn, "BRIGHT lights, BIG city: high redshift radio galaxies, giant Ly-a halos, and proto-clusters," in Discoveries and Research Prospects from 6- to 10-Meter-Class Telescopes II. Edited by Guhathakurta, Puragra. Proceedings of the SPIE, Volume 4834, pp. 24-34 (2003)., pp. 24-34, Feb. 2003.

6. P. L. Shopbell, S. Veilleux, and J. Bland-Hawthorn, "The Very Extended Ionized Nebula around the Quasar MR 2251-178," ApJL 524, pp. L83-L86, Oct. 1999.

7. S. A. Stanford, R. Elston, P. R. Eisenhardt, H. Spinrad, D. Stern, and A. Dey, "An IR-Selected Galaxy Cluster at $\mathrm{z}=1.27, " A J \mathbf{1 1 4}$, pp. 2232-+, Dec. 1997.

8. P. G. van Dokkum and S. A. Stanford, "A Massive Disk Galaxy at z=1.34," ApJL 562, pp. L35-L38, Nov. 2001.

9. C. C. Steidel, K. L. Adelberger, M. Dickinson, M. Giavalisco, M. Pettini, and M. Kellogg, "A Large Structure of Galaxies at Redshift Z approximately 3 and Its Cosmological Implications," ApJ 492, pp. 428-+, Jan. 1998.

10. C. C. Steidel, K. L. Adelberger, A. E. Shapley, M. Pettini, M. Dickinson, and M. Giavalisco, "Ly $\alpha$ Imaging of a Proto-Cluster Region at $<\mathrm{z}>=3.09, " A p J$ 532, pp. 170-182, Mar. 2000.

11. J.-G. Cuby, O. Le Fèvre, H. McCracken, J.-C. Cuillandre, E. Magnier, and B. Meneux, "Discovery of a z = 6.17 galaxy from CFHT and VLT observations," A $\& A$ 405, pp. L19-L22, July 2003.

12. J. E. Rhoads, S. Malhotra, A. Dey, D. Stern, H. Spinrad, and B. T. Jannuzi, "First Results from the Large-Area Lyman Alpha Survey," ApJL 545, pp. L85-L88, Dec. 2000.

13. J. E. Rhoads, A. Dey, S. Malhotra, D. Stern, H. Spinrad, B. T. Jannuzi, S. Dawson, M. J. I. Brown, and E. Landes, "Spectroscopic Confirmation of Three Redshift z 5.7 Ly $\alpha$ Emitters from the Large-Area Lyman Alpha Survey," AJ 125, pp. 1006-1013, Mar. 2003. 